\title{
ESTRUCTURA Y DINAMICA DEL PLANCTON EN LA LAGUNA DE GALLOCANTA DURANTE 1981
}

X.Rodó y F.A.Comín

Dep.d'Ecologia, Fac.Biologia, Universitat de Barcelona.

Palabras clave: zooplancton, fitoplancton, diversidad, sucesión, escalas, salinidad.

Zooplankton and phytoplankton data from Lake Gallocanta, NE Spain, were examined to determine the structure and changes in the community patterns. Diversity values (Shannon-Wiener $H$ index) move between 1.93 bits and 0.01 bits for phytoplankton and are under 1 for zooplankton during the whole period. Armstrong succession index shows how both communities change at a rate faster than the sampling scale used here. It is concluded that determining the role salinity and time play is fundamental to the understanding of processes in saline lakes.

\section{INTRODUCCION}

La organización de las comunidades biológicas en los ecosistemas es función de la intensidad y frecuencia de las perturbaciones que los afectan. En ecosistemas muy fluctuantes, las perturbaciones impiden a las comunidades sobrepasar las etapas iniciales de la sucesión y el control del funcionamiento del ecosistema es ejercido por factores extrínsecos al mismo (Margalef, 1991).

No obstante, la apreciación del grado de organización o estructura de una comunidad y más importante aún, de su tasa de cambio respecto al tiempo, es función de la escala de tiempo utilizada en la observación (Comin et al, 1992).

En los lagos salinos, la baja estructuración de las comunidades ha sido repetidamente justificada como consecuencia sobre todo, de los cambios de salinidad (Hammer, 1986). Pero una apreciación detallada en el tiempo y de los diversos componentes de la comunidad, puede ofrecer detalles de cambios en la estructura de la comunidad, que revelen procesos de organización de distintos niveles tróficos de la comunidad a diferentes escalas de tiempo. Los datos y tratamiento que se ofrecen aquí sobre la laguna de Gallocanta (Aragón, NE. España), sirven para ilustrar estas ideas.

\section{MATERIAL Y METODOS}

La estructura de la comunidad planctónica se estudió a partir de datos de recuentos de zooplancton y fitoplancton de 1980-81 (Comin et al., 1983), de la laguna de Gallocanta (Aragón). Asímismo se utilizaron los datos de nutrientes que aparecen on el mismo artículo.

Como descriptor del grado de organización de la comunidad, se utilizó el índice de diversidad de Shannon-Wiener (1949), $\left(H=-\Sigma\left(p_{1}^{*} \ln \left(p_{i}\right)\right)\right.$, donde $p_{1}$ es la probabilidad que una especie $i$ aparezca en una muestra), mientras que para estimar el cambio en la estructura temporal, se ha usado el índice de sucesión de Armstrong (1969), que 
mide como cambia la contribución de las diferentes especies a la diversidad. Asi tenemos:

$$
S_{b b}=\left(\sum\left(\frac{f_{b}-f_{b}}{b-a}\right)^{2}\right)^{1 / 2}
$$

en donde $\mathrm{S}_{\text {nh }}$ es la tasa diaria de variación de la comunidad en el espacio de diversidad, $(b-a)$ es el intérvalo de tiempo en dias y $f_{\text {ia }}$ y $f_{i b}$ son las contribuciones de la especie i a la diversidad en los tiempos a y b, de manera que :

$$
f_{\text {ti }}=\frac{\frac{x_{\text {ba }}}{x_{a}} \log \frac{x_{\text {la }}}{x_{a}}}{H_{a}}
$$

$x_{\text {la }}$ es el $n^{8}$ de organismos de la especie i en el tiempo $a, x_{a}$ es el $n^{8}$ total en dicha fecha y $\mathrm{H}$ es la diversidad en el tiempo a.

\section{RESULTADOS Y DISCUSION}

Durante 1981, se inició una fase de vaciado de la laguna de Gallocanta. EI nivel del agua descendió de 95 a $48 \mathrm{~cm}$. y la cantidad de sales disueltas pasó de 42 a $103 \mathrm{~g} / \mathrm{l}$ (Fig. 1).

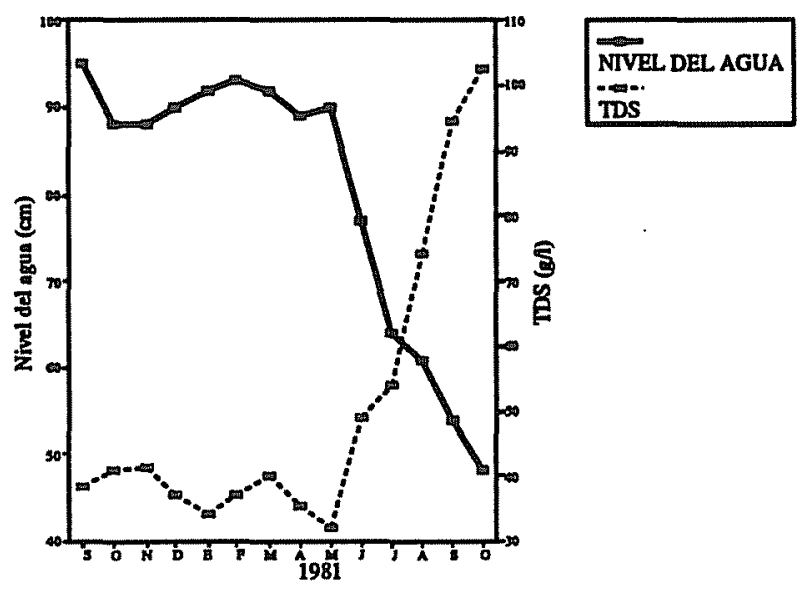

Fig.1. Variaciones del nivel del agua y de los sólidos totales disueltos.

Los valores de diversidad $(H)$ para el fitoplancton, fueron superiores a los del zooplancton en casi todo el período considerado, excepto entre Abril y Julio. H osciló entre 1.93 bits/ind en Febrero y 0.01 en Mayo de 1981, mientras que los valores fueron inferiores a 1 para el zooplancton durante todo el muestreo. Estos valores, tan 
relativamente bajos comparados con otros ecosistemas (Margalef, 1991), son similares a los descritos para otros lagos salados (Hammer, 1986).

El zooplancton y el fitoplancton muestran dinámicas opuestas, en lo que respecta a la evolución temporal del grado de organización de las dos comunidades. Se pueden distinguir fases diferentes en la diversidad del fitoplancton (Fig.2). Una primera, que iría de Septiembre de 1980 a Febrero de 1981, en la cual $\mathrm{H}$ aumenta y se alcanzan los valores máximos. Durante este inténvalo de tiempo la salinidad no varía significatívamente. De Febrero a Mayo, se produce una disminución muy notable de la diversidad, hasta valores casi nulos $(H=0.01)$, debido a que el fitoplancton está mayoritariamente constituido por una sola especie (Lobocystis dichotoma, $p_{l}=0.99$ ). A partir de entonces, la diversidad presenta una tendencia creciente hasta Octubre de 1981. Las apariciones/desapariciones de especies en las muestras, se suceden con una frecuencia, en muchos casos superior a la mensual. Esto no se aprecia en la representación de las células del fitoplancton con respecto al tiempo (Fig.3), debido a que la escala de muestreo no alcanza a detectar dichas fluctuaciones en las poblaciones. De hacerlo, éstas se reflejarian como dientes de sierra u ondas de lambda inferior a 1 mes. El número de células fitoplanctónicas por $\mathrm{ml}$, parece tener un crecimiento con asintota en $3^{\star} 10^{5}$ (Fig.3).

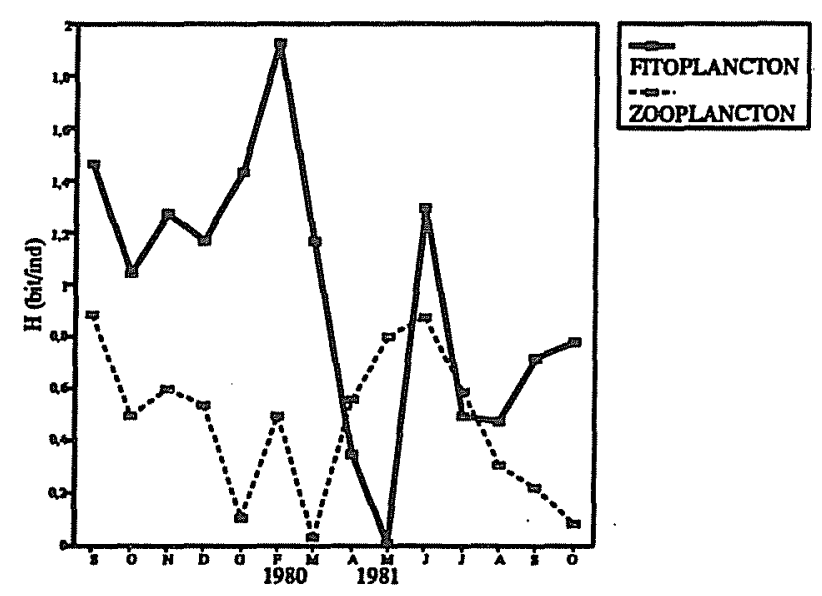

Fig 2: Variaciones de la diversidad para el zooplancton y el fitoplancton durante 1981.

La salinidad y el tiempo, pueden llegar a convertirse en ejes organizadores importantes, en lagos salinos altamente fluctuantes. En lo que respecta a la salinidad, su efecto en las poblaciones difiere según la época considerada, ya que p.e. de Septiembre de 1980 a Mayo de 1981, no cambia significativamente, mientras que a partir de dicha fecha, va incrementándose paulatinamente hasta valores superiores a los $100 \mathrm{~g} / \mathrm{l}$. Durante el primer período, la comunidad fitoplanctónica se estructura de manera que sus valores de $H$ van creciendo. Entre Diciembre y Enero, se detectan concentraciones relativamente altas de $\mathrm{N}-\mathrm{NO}_{3}$ y de SRP (Comín et al, 1983). Con un 
desfase de poco más de un mes aproximadamente -imprecisión ésta, impuesta por la escala de muestreo-, la H fitoplanctónica desciende hasta los valores antes citados de 0.01 . A partir de este momento y a medida que aumenta la salinidad, las especies del fitoplancton se suceden muy rápidamente -a menudo con una frecuencia superior a la de muestreo-. La segunda vez que se detectan valores elevados de nutrientes Mayo y Junio de 1981- el efecto sobre la comunidad es distinto, porque las condiciones de salinidad han variado ostensiblemente.

Podriamos decir que sobre el eje de la salinidad, se irian superponiendo otros ejes -organizadores o desorganizadores, según la escala temporal que se considerey que corresponderían a las pertubaciones exógenas, que tienen un efecto variable dependiendo de la posición -en un momento dado- del sistema sobre el eje de salinidad.

El tiempo es también, en estos ambientes altamente fluctuantes, una variable estructural importante, tanto por lo que se refiere a aspectos metodológicos (como la escala de muestreo), como por su importancia a la hora de interpretar los procesos que se producen en la laguna y los ciclos en los que todo el sistema se mueve.

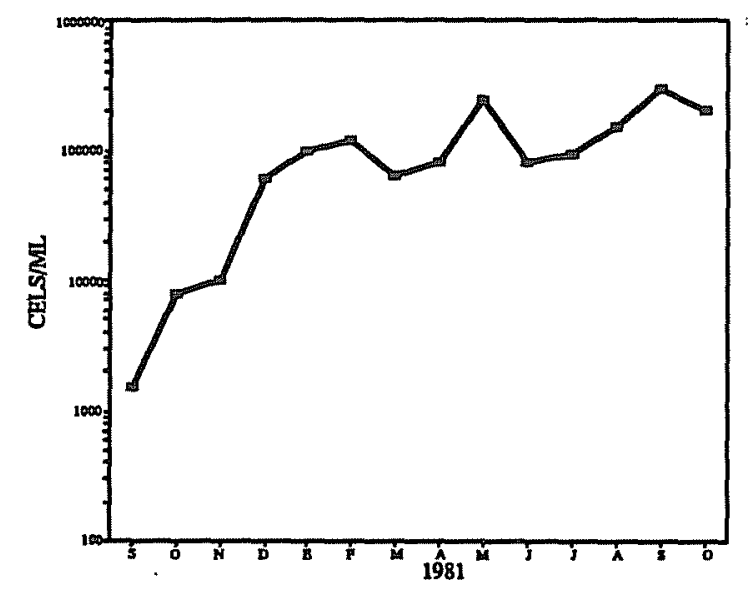

Fig.3: Variaciones mensuales de la densidad media total del fitoplancton en la laguna de Gallocanta durante 1981.

\section{Tasa de cambio $\left(S_{\mathrm{ab}}\right)$}

El sistema está caracterizado por los pulsos en las tasas de cambio de ambas comunidades, que responden a una aparición/desaparición más o menos continuada de especies en el tiempo y la frecuencia de este proceso va variando según como evolucione la salinidad, tanto en lo que hemos dado en llamar fases diferentes, como según cuál sea la "macrojase" en las que éstas estan englobadas (Comin et al.,1991).

La percepción de la tasa de cambio del fitoplancton en base a estos datos, topa con la limitación impuesta por la escala de muestreo que se utilizó, que no sirve para poner de manifiesto cambios en las poblaciones planctónicas, de sistemas con elevada frecuencia de fluctuación -en este caso, inferior a un mes, que fué la escala 
temporal de recolección de muestras. Con el intervalo mensual, estamos involuntariamente efectuando un "alisado" de la serie de cambios intramensuales.
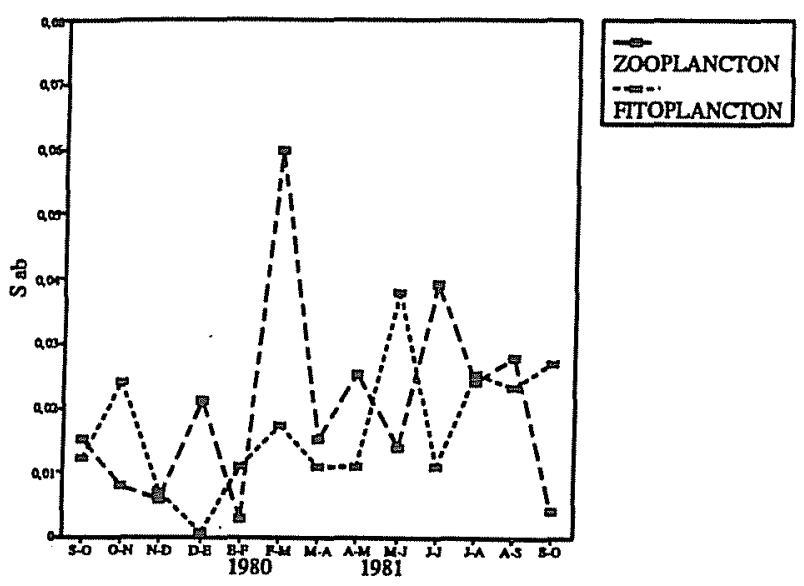

Fig.4: Variaciones del indice de sucesión de Armstrong.

El valor mínimo de la tasa de cambio del fitoplancton se da entre Diciembre y Enero (Fig.4), mientras la diversidad muestra una tendencia ascendente(Fig.3). El máximo se observa entre Mayo y Junio. Para el zooplancton en cambio, en los meses de Febrero y Marzo, $S_{a b}$ adquiere el valor máximo, coincidiendo con una disminución considerable en las diversidades de ambas comunidades. Se intuye aquí también como para el caso de la diversidad-, que productores y consumidores muestran dinámicas inversas, sobre todo a partir de Abril.

No obstante, deben tenerse en cuenta algunas consideraciones. La tasa de cambio aparece sobreestimada para el zooplancton, con respecto al fitoplancton, porque en el primer caso hay pocas especies. En consecuencia, la ausencia/presencia y/o el cambio en la abundancia de una especie, tiene más peso que en el caso del fitoplancton. Lo mismo pasa para la $\mathrm{H}$. Es decir, que la comparación entre las dos comunidades se podría hacer únicamente, a nivel de tendencias generales, puesto que aunque las tasas $\left(\mathrm{S}_{\mathrm{ab}}\right)$ vengan expresadas en las mismas unidades, las escalas no serian totalmente comparables.

Finalmente, se han representado los valores de las frecuencias relativas de las diferentes especies $\left(P_{1}\right)$ para las fechas de máxima y minima diversidad, ponderadas con respecto a los rangos que dichas especies tienen en cada muestra (Fig.5). De este modo, la pendiente que se obtiene en cada caso, es indicativa del grado de organización de la comunidad. Se observa también una discordancia en el tiempo, entre los grados de organización del fito- y del zooplancton. 


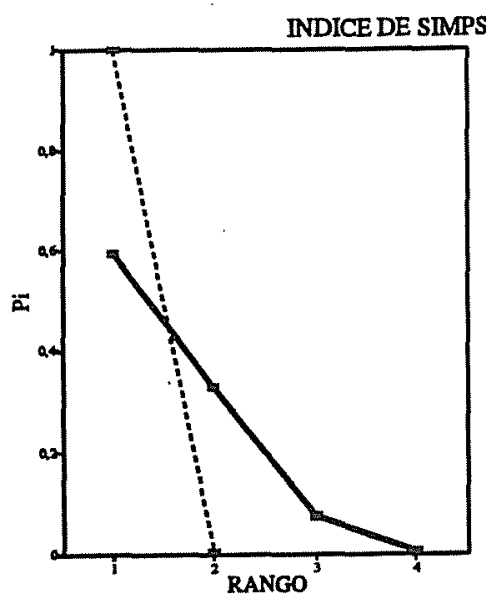

ZOOPLANCTON(JULO)
ZO...
ZOOPLANCTON(MARZO)

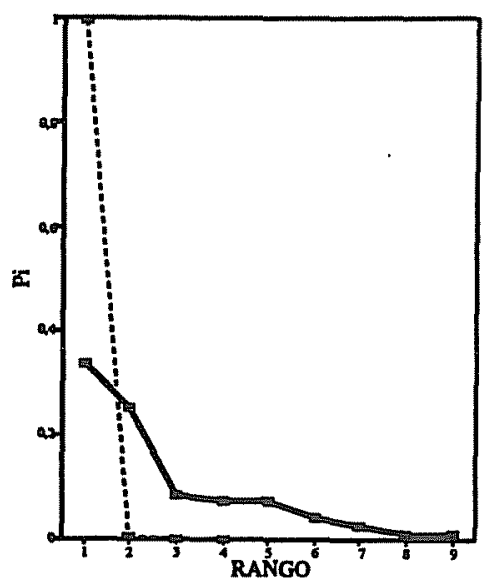

FTMOPLANCTON(FEBR) FITOPLANCTON(MAYO)

Figs. 5 y 6: Frecuencias relativas de las especies de fitoplancton y zooplancton, respecto de los rangos que ocupan en dichas muestras, en las épocas de mayor y menor diversidad.

\section{CONCLUSIONES}

Las comunidades planctónicas de la laguna de Gallocanta cambian rápidamente de estructura a lo largo del tiempo. La determinación de las tasas de cambio (Sab) de las poblaciones que las componen, exige acoplar la escala de muestreo a la frecuencia de dicho cambio.

Los valores de diversidad(H) del fitoplancton y del zooplancton, presentan evoluciones inversas. La diversidad del fitoplancton oscila entre 0.01 y 1.93 , mientras que no se alcanza el valor de 1 para el zooplancton, en todo el muestreo.

La correcta apreciación del papel primordial que juegan el tiempo y la salinidad, es fundamental para explicar como ocurren los cambios en la organización de la estructura trófica de este tipo de ecosistemas.

\section{AGRADECIMIENTOS}

Este estudio fué financiado por la Diputación General de Aragón, Servicio de 
Conservación del Medio Natural. X.R. es becario de F.P.I. del Ministerio de Educación y Ciencia.

\section{BIBLIOGRAFIA}

Armstrong, R. 1969. Phytoplankton Community Structure in Castle Lake, Califomia. Ph.D thesis. Univ. of Califomia, Davis. 149 pp.

Comin, F.A., M.Alonso, P.Lopez \& M.Comelles, 1983. Limnology of Gallocanta Lake, Aragon, northeastem Spain. Hydrobiologia 105: 207-221.

Comin, F.A., R.Julià \& M.P.Comin, 1991. Fluctuations, the key aspect ior the

ecoloqical interpretation of saline lake ecosystems. Oecologia Aquatica, 10: 127-135. Comin, F.A., X.Rodó \& M.P.Comín, 1992. Lake Gallocanta (Aragón, NE. Spain), a paradiam of fluctuations at different time scales. Limnetica 8:79-86. Symposium. Hammer, U.T., 1986. Saline Lake Ecosystems of the World. Junk, The Hague, 602 pp. Margalef, R., 1991. Teoria de los sistemas ecológicos. Estudi General 1. Publi. Univ. Barcelona.

Shannon, C.I. \& W.Wiener, 1949. The mathematical theory of communication. Illinois Books, Urbana. 